\title{
La presse française commente la question macédonienne à l'aube des guerres balkaniques
}

\section{Nicolas Pitsos}

\section{OpenEdition}

12 Journals

Édition électronique

URL : https://journals.openedition.org/ceb/846

DOI : $10.4000 /$ ceb.846

ISSN : 2261-4184

Éditeur

INALCO

\section{Édition imprimée}

Date de publication : 30 mars 2011

Pagination : 181-196

ISBN : 978-2-85831-189-7

ISSN : 0290-7402

\section{Référence électronique}

Nicolas Pitsos, "La presse française commente la question macédonienne à l'aube des guerres balkaniques », Cahiers balkaniques [En ligne], 38-39 | 2011, mis en ligne le 05 décembre 2011, consulté le 06 juillet 2021. URL : http://journals.openedition.org/ceb/846 ; DOI : https://doi.org/10.4000/ceb. 846

Ce document a été généré automatiquement le 6 juillet 2021.

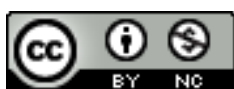

Cahiers balkaniques est mis à disposition selon les termes de la Licence Creative Commons Attribution - Pas d'Utilisation Commerciale 4.0 International. 


\title{
La presse française commente la question macédonienne à l'aube des guerres balkaniques
}

\author{
Nicolas Pitsos
}

1 Cette étude s'inscrit dans un projet d'élargissement de l'historiographie de la question d'Orient ${ }^{1}$. Dans cette perspective, j'entends aborder les événements composant cette fresque historique, par une voie peu exploitée jusqu'à maintenant, à travers leurs échos auprès de la société française. Pour ce faire, je m'intéresse surtout aux jugements et aux raisonnements des principaux formateurs de l'opinion publique à cette époque, et en premier lieu, aux journaux.

2 Dans ma communication, je me suis assigné comme objectif, d'analyser l'une des séquences les plus caractéristiques de la question d'Orient, celle qui met la Macédoine et la question macédonienne au début de la Première Guerre balkanique, au centre des discours et des enjeux politiques. Ma méthode est celle employée en histoire des représentations, dans le sens que ceux qui pratiquent l'histoire socioculturelle lui ont accordé, c'est-à-dire l'étude de la manière dont les individus, dans un espace donné et pendant une période précise, conçoivent le monde autour d'eux et se le représentent ${ }^{2}$.

3 En ce qui me concerne, j'ai voulu comprendre comment les Français de cette époque ont pu se représenter la scène sur laquelle se confronteraient les acteurs du spectacle guerrier auquel ils allaient assister grâce aux rapports des envoyés spéciaux des journaux. Quelles ont été leurs interprétations et leur compréhension des prolégomènes et du premier acte de cette trame? En quoi leurs jugements sur l'issue de cette tragédie ont-ils varié en fonction de leurs sympathies envers tel ou tel autre protagoniste et selon leurs propres convictions idéologiques?

4 Un des outils fondamentaux pour l'écriture d'une telle histoire étant la consultation de la presse, cette véritable et redoutable machine de production de représentations, les sources que j'ai explorées pour cette communication comprennent surtout des journaux. Quant à la période choisie, il s'agit du mois d'octobre 1912, débutant quelques jours avant et finissant quelques jours après la proclamation officielle de la Première 
Guerre balkanique, le 16 octobre ${ }^{3}$. Les journaux retenus dans ma recherche couvrent un large spectre idéologique et témoignent de sensibilités politiques diverses et variées ${ }^{4}$. Il s'agit des quatre grands quotidiens populaires de l'époque, Le Petit Journal (républicain puis de droite), Le Petit Parisien (républicain), Le Matin (républicain modéré), Le Journal (républicain puis de droite), des journaux regroupant les différentes tendances de la droite française ${ }^{5}$, Le Figaro, Le Journal des Débats, Le Gaulois, La Croix (droite catholique), L'Écho de Paris, L'Éclair (droite nationaliste), l'Autorité (bonapartiste), Le Temps, et des journaux réunissant différentes expressions de la gauche, notamment le journal officiel du Parti radical, Le Radical, et la voix socialiste, avec L'Humanité, mais également $L a$ Petite République. J'ai croisé et complété ce corpus avec des mémoires publiés sur la région de Macédoine et avec des essais portant sur le fonctionnement de la presse française vu par les contemporains.

\section{La Macédoine et la question macédonienne vues par les journalistes français}

\section{Les représentations géographiques}

5 À l'aube de la Première Guerre balkanique, la Macédoine est toujours considérée comme une région exotique, une contrée du continent européen peu visitée, d'où la nécessité d'éduquer le public, de l'instruire, à l'aide des cartes et autres documents imprimés, distribués par les journaux, en supplément, ou intégrés aux éditions quotidiennes. Les événements qui agitent et mettent le feu à la péninsule balkanique semblent susciter un intérêt considérable parmi les citoyens français de l'époque, comme le rapporte le rédacteur du Gil Blas:

\footnotetext{
"À Paris, il n'est plus qu'un sujet d'entretien : la guerre. Métro, autobus, salon, antichambre, et l'office et l'alcôve ne parlent plus que des Balkans. C'est un commun délire. À tout coin de rue, des prophètes vous accostent et vous assomment de leurs jérémiades; des diplomates au pied levé vous importunent de leur dernier tuyau; [...] le piquant dans l'affaire,c'est qu'aucun de ces gobe-mouches ne sait précisément si c'est Belgrade, la capitale de la Serbie, ou Sophia, ou Bucharest ou Philipoppoli ou Pampelune! Dans leur émoi, ces bonnes gens confondent volontiers Valaques, Roumains, Croates, Bulgares et vous en font la plus étrange Macédoine du monde. [...] $»^{6}$
}

6 Si le commun des mortels ignorait presque tout des contours de cette région de l'Empire ottoman, les experts de cette question ne parvenaient pas, eux non plus, à en délimiter les frontières. Gabriel d'Azambuja, fidèle disciple de l'école leplaysienne ${ }^{7}$ et consciencieux héritier de ses traditions sociologiques, nous livre une monographie sur une ville macédonienne au début du XXe siècle. Dans cet ouvrage, il avoue que la Macédoine elle-même est une expression géographique au sens assez vague, correspondant à un territoire dont les limites n'ont jamais été bien fixées. ${ }^{8}$

7 La définition des frontières de cette région varie en effet selon les auteurs et leurs considérations personnelles. On pourrait dire qu'il y avait au moins deux perceptions de cet espace. Une définition européenne, forgée au XIX ${ }^{e}$ siècle, et qui correspondrait au tracé très géométrique du journaliste du journal Le Temps, selon lequel :

"[...] La Macédoine [...] occupe sur la carte la surface d'un triangle rectangle dont

l'hypoténuse s'étendrait de l'extrémité méridionale du Pinde, à Metzovo, jusqu'à

l'embouchure de la Maritza, à Dédéagatch ou à Enos. Le sommet de l'angle droit se place à 
peu près à la hauteur de la ville de Varosh, [...], à mi-chemin d'Uskub et de Mitrovitza, non

loin des sources du Vardar. "» et qui est présentée par le journaliste français Henry Spont, dans La Nouvelle Revue de 1903, quand il précise que la "Macédoine» (terme qui, en revanche, n’a jamais été employé comme tel dans la nomenclature ottomane) est une expression géographique qui comprend les vilayets de Salonique, de Monastir et une partie du vilayet de Kosovo. Quelques années plus tard, en 1912, son collègue du journal Le Temps, considère qu'une partie du vilayet d'Andrinople en fait également partie.

\section{Les lectures-appropriations historiques}

9 Malgré l'incertitude de la définition des limites spatiales de la Macédoine, cette région, en tant que division territoriale de l'administration ottomane, était le point de convergence des irrédentismes de tous les royaumes voisins des domaines du sultan. Les journalistes français de l'époque semblent en être pleinement conscients. C'est ainsi que Léo Clarétie écrit, dans Le Petit Parisien du 12 octobre $^{10}$, que la mobilisation en cours à Sofia, n'est que «l'effort d'un peuple qui veut par-dessus les âges reprendre toute sa place au soleil, et qui lutte pour la plus grande Bulgarie, les yeux fixés sur la Macédoine. [...] »

acédoine faisait partie du projet d'une Grande Bulgarie, qui renvoyait à l'étendue territoriale des royaumes médiévaux des tsars Siméon et Samuel ou à celle de la dynastie des Asanides $^{11}$ et dont la réincarnation au XIX ${ }^{e}$ siècle, ne serait-ce qu'éphémère, était due aux dispositifs stipulés par le Traité de San Stefano en $1878^{12}$.

11 De leur côté, les Serbes nourrissaient, eux aussi, depuis l'époque de l'élaboration de Nacertanje $^{13}$, le rêve de reconstituer l'empire de Dušan, qui comprenait la Macédoine, comme le rappelle André Morizet à ses lecteurs, dans L'Humanitée ${ }^{14}$.

12 Cette région faisait également partie de la Grande Idée grecque (Megali Idea) ${ }^{15}$, volonté de l'État grec de reconstituer l'Empire byzantin. La « légitimité » d'un tel projet était en quelque sorte cautionnée par certains commentaires comme celui de l'envoyé spécial du journal Le Matin ${ }^{16}$ qui écrit le 26 octobre que : «Pour la première fois depuis 450 ans, un prince grec a pénétré en Macédoine à la tête de son armée »

On constate dans cette affirmation un amalgame très courant dans l'historiographie du $\mathrm{XIX}^{\mathrm{e}}$ et même du $\mathrm{XX}^{\mathrm{e}}$ siècle, qui consiste à assimiler l'Empire Romain d'Orient, autrement connu sous le terme anachronique d'Empire byzantin, à un royaume grec. Le calcul chronologique de ce commentaire montre aussi à quel point les différentes étapes de stratification historique de cet espace n'étaient pas forcément connues par tous les journalistes de l'époque. En effet, la Macédoine, dans son acception européenne du XIX ${ }^{e}$ siècle, avait cessé d'appartenir à l'Empire byzantin au moins un siècle avant la prise de Constantinople par les Turcs ottomans et leurs alliés, événement auquel l'envoyé spécial du Matin fait allusion.

\section{Les estimations ethnologiques}

En dehors de l'inclusion de cet espace dans les desseins et projets expansionnistes des royaumes de la péninsule balkanique, au nom d'une lecture partielle et partiale de l'histoire, un autre aspect conflictuel de cette contrée de l'Empire ottoman réside dans sa composition ethnique. Encore une fois, les journalistes français de cette période sont 
particulièrement avertis de la complexité de la situation. Ainsi Jules Arren, du journal L'Éclair, intitule son article consacré au commentaire de la crise dans les Balkans, le casse-tête macédonien. Ce qui rend, selon lui, presque insoluble l'imbroglio macédonien, ce sont les Macédoniens : « [...] Il y a dans ce pays un tel enchevêtrement de races, de religions et de langues, que tout partage, toute émancipation et tout gouvernement soulèvent aussitôt des objections formidables. ${ }^{17}$ Il finit sa présentation de la situation prévalant en Macédoine, au début du conflit balkanique, en constatant que la Serbie, la Grèce, la Bulgarie et la Roumanie se disputent ce malheureux pays à coups de statistiques, sans avoir encore pu se mettre d'accord. "Chacun y fait sa propagande, au moyen d'écoles, de bandes armées, ne faisant qu'accroître la confusion. $»^{18}$

\section{La question macédonienne à l'origine de la Première Guerre balkanique}

Les approches divergentes et contradictoires de la configuration géographique, de la composition ethnique et de l'appartenance historique de cet espace, posent les termes de la question macédonienne, à savoir du destin de cette région de l'Empire ottoman, dans un contexte d'antagonismes impérialistes ${ }^{19}$ et d'exaspérations nationalistes. $\mathrm{Au}$ mois d'octobre 1912, le journaliste de La Croix affirme que :

"[...] c'est la question de la Macédoine, qui depuis une quinzaine d'années conditionne la politique extérieure et intérieure de la Turquie. C'est la question de la Macédoine à peine généralisée qui motive aujourd'hui le conflit balkanique. [...] ${\aleph^{20}}^{2}$

En effet, au moins depuis 1903 et la révolte d'Ilinden, événement qui marque l'apogée de l'activisme politique et militaire de l'ORIM (Organisation révolutionnaire intérieure macédonienne), la Macédoine préoccupe constamment les autorités ottomanes et occupe une place importante dans leur politique intérieure et leurs relations avec les grandes puissances ${ }^{21}$.

\section{Typologie des interprétations des causes du conflit}

Si la presse est unanime sur les origines de la Première Guerre balkanique et la place de la question macédonienne dans celle-ci, des approches divergentes apparaissent toutefois au sujet des motivations réelles des acteurs impliqués dans cette confrontation.

Une partie des journalistes adopte la position officielle des gouvernements des pays balkaniques, qui déclarent se battre pour la protection des droits de leurs coreligionnaires. D'autres voient la mobilisation des alliés comme la volonté d'une expansion territoriale au détriment de l'Empire ottoman, en vue de la réalisation des Grandes Idées respectives. Dans une telle optique, ils y décèlent également, derrière les ambitions démesurées des royaumes balkaniques, les traces des rivalités entre grandes puissances. 


\section{Le conflit vu comme prétexte pour des conquêtes inavouées : une approche géopolitique - marxiste} d'Orient par les socialistes des Balkans ${ }^{24}$. Marcel Cachin nous informe que ses camarades ont essayé de montrer à leurs concitoyens que le but des nationalistes de leur pays n'était pas de réclamer, comme ils le disaient, plus de liberté pour les chrétiens d'Orient, mais de convoiter des marchés «pour le placement de leurs marchandises, de leurs capitaux et de l'excès de leur personnel bureaucratique ». Ce qu'ils recherchent, ajoute le futur directeur de L'Humanité, c'est, à l'exemple de tous les pays capitalistes petits et gros, leur expansion territoriale et économique. Et il conclut son analyse en affirmant que «ce qui s'agite en Orient présentement, les causes essentielles de la guerre sauvage qui va déshonorer, une fois de plus, notre humanité qui se croit civilisée, ce sont $[. .$.$] les intérêts. »^{25}$

\section{Le conflit vu comme volonté de protection de populations menacées : une approche culturaliste - raciste}

Ces raisonnements étaient taxés de lourd matérialisme par une partie de la presse, Le Temps en tête, ce journal de la bourgeoisie républicaine, qui considère que, derrière la mobilisation des alliés contre l'Empire ottoman, il y a un souci de protection des populations, notamment en Macédoine.

Avant même que la Première Guerre balkanique n'éclate, le Journal des Débats justifie l'agitation belliqueuse dans la région par un mécontentement inspiré par la politique violente de «turquification » qu'a suivi l'arrivée au pouvoir du parti Jeune-Turc, et qui a remplacé l'apaisement qui existait sous l'effet des promesses de l'ottomanisme libéral de la révolution de 1908. Depuis, tout n’a plus été que déception pour les chrétiens de Macédoine ${ }^{26}$.

Imprégnés de la même conviction, des journaux comme Le Gaulois ${ }^{27}$, faisant écho à la presse royaliste, présentent la démarche agressive des alliés comme un effort de délivrance de frères opprimés, tandis que $\mathrm{La} \mathrm{Croix}^{28}$, carillonnant la voix catholique du journalisme français, compare l'opération des alliés aux croisades médiévales.

Cahiers balkaniques, 38-39 | 2011 

couverture par les journaux de l'escalade de la question macédonienne vers une confrontation militaire. Louis Dubreuilh constate, à son grand déplaisir, que tous les journaux, ou presque tous les journaux de la presse militariste et chauvine font l'éloge des alliés balkaniques contre les Turcs. ${ }^{34}$

31 En revanche, le rédacteur de La Croix est étonné par les sympathies dont on entoure les Turcs. Selon lui, une campagne systématique est menée à Paris en leur faveur, avec la Bourse, l'ambassade d'Autriche-Hongrie et certains bureaux du quai d'Orsay, comme bases d'opérations ${ }^{35}$. Derrière cette campagne turcophile, La Croix voit aussi l'ambassade ottomane qui "aurait largement entamé ses 'fonds de presse' et généreusement arrosé les journaux qui ont accepté ses inspirations $»^{36}$.

Opposés aux tenants de l'approche géopolitique et marxiste, pour qui les mobiles économiques et les intérêts politiques prévalaient dans l'explication du déclenchement de la Première Guerre balkanique, les partisans de ce que j'appelle une approche culturaliste et raciste mettent en avant des causes de modèle « civilisationnel ${ }^{29}$ ou d'appartenance raciale. Comme le fait remarquer Louis Dubreuilh, dans L'Humanité, pour les apôtres de ce point de vue: «C'est une lutte de race et de religion qui s'est engagée: Aryens contre Touraniens, chrétiens contre musulmans, Croix contre Croissant. [...] $»^{30}$ Dans un tel discours, les alliés sont censés représenter la civilisation alors que les Ottomans symbolisent la barbarie. ${ }^{31}$

l'opinion publique sur les causes du conflit armé qui allait secouer l'Empire ottoman, explique à ses concitoyens que : «si les chrétiens des Balkans vont se confronter aux Turcs, c'est moins par l'effet de la différence des races, qu'en raison d'une diversité de civilisations qu'une expérience prolongée a montrée radicalement inconciliables. $»^{32}$

des questions étrangères du Journal inscrit la lutte turco-balkanique dans «le conflit gigantesque qui met aux prises le christianisme et l'islam depuis plus de mille ans. C'est le choc de deux conceptions morales, de deux civilisations, de deux races. C'est une guerre nationale et religieuse. $»^{33}$ représentations de l'Autre, en l'occurrence du musulman et du Turc, dans une partie de la société française du début du XXe siècle ou, pour être encore plus précis, permettent de percevoir la direction vers laquelle une partie de l'opinion publique aurait pu évoluer si elle s'était laissé manipuler par de tels jugements.

\section{Déclinaison des sympathies de la presse française à l'égard des acteurs de la Première Guerre balkanique}

Ces joutes journalistiques et les tensions entre les différents représentants de la presse française $e^{37}$, tout en mettant le doigt sur des pratiques douteuses de financement ${ }^{38}$, sont en même temps, révélatrices d'un contexte sociopolitique qui se prêtait parfaitement, me semble-t-il, à une polarisation de la société autour de deux camps opposés. Après le coup d'Agadir, en 1911, les passions nationalistes ${ }^{39}$ étaient de nouveau agitées et ravivées en France. La décision du gouvernement de Berlin, d'accroître les dépenses militaires et les débats à l'Assemblée sur une éventuelle modification de la loi sur la 
conscription militaire, qui sera finalement votée par le cabinet d'Aristide Briand au mois de juillet 1913, portant la durée du service militaire de deux à trois ans ${ }^{40}$, n'ont fait pour le reste que creuser le clivage entre pacifistes et militaristes. Enfin, les relations diplomatiques de la République et notamment, son alliance avec la Russie, relevaient également d'une question d'intérêt national et divisaient davantage la presse française.

D'autre part, la loi de séparation de l'Église et de l'État constituait toujours une pierre d'achoppement à la normalisation des relations entre partisans d'un catholicisme intransigeant et ténors d'une libre-pensée anticléricale ${ }^{41}$ et fournissait un moyen de contestation majeure à tous ceux qui étaient plus ou moins ouvertement hostiles à la République laïque.

Jean Jaurès trace dans L'Humanité les lignes de démarcation idéologiques des deux tendances politiques et des deux sensibilités philosophiques aux prises pendant les premiers jours du conflit balkanique. Il pense que les représentants de la réaction, aussi bien à l'extérieur qu'à l'intérieur de la République, c'est-à-dire les dynasties impériales autrichienne et russe, d'une part, et les forces chauvines et cléricales, d'autre part, exploiteront l'effondrement de la Turquie nouvelle, contre toute idée de paix, de démocratie et de pensée libre. ${ }^{42}$

En ce qui nous concerne, on pourrait distinguer trois grandes approches de la crise balkanique et trois groupes respectifs de journalistes voire de journaux.

Il y a ceux qui œuvrent pour la paix et sont critiques à l'égard des intentions belliqueuses des alliés tout en optant pour la mise en place d'une fédération des peuples dans les Balkans et notamment en Macédoine.

Ensuite, il y a ceux qui, soit par sympathie envers les Ottomans, soit par la crainte d'un recul de l'influence des missions catholiques soutenues par la France et d'une éventuelle prédominance orthodoxe en Macédoine, souhaitent le maintien du statu quo territorial.

Enfin, il y a ceux qui s'expriment en faveur des tentatives des souverains des royaumes chrétiens et qui se montrent hostiles aux Ottomans et à leur présence dans le continent européen.

\section{En faveur de la paix : l'idée de la création d'une fédération}

En adoptant la première position, le journal Gil Blas publie, le 5 octobre, le discours qu'Anatole France prononça à la mémoire d'Émile Zola, à l'occasion de la commémoration des dix ans sa disparition. Dans cet hommage, il affirma que :

"À cette heure où en Europe, et dans le monde entier, toute idée de justice et de droit est foulée aux pieds, où les peuples n'ont qu'un seul culte, la force, un seul idéal, la violence, où l'on est traité de mauvais citoyens dès qu'on s'oppose aux entreprises dirigées par la brutalité ou la cupidité contre la paix universelle et les libertés publiques, il convient de rappeler ce que fit Zola, il y a quatorze ans. [...] délibérément, il fit le sacrifice de sa popularité, de son repos, de son travail, et se jeta dans les fatigues et les périls pour éviter une injustice à son pays et se montrer lui-même un juste. $»^{43}$

Dans l'ambiance de fanatisme religieux prôné par une partie de la presse française qui prêche la croisade contre les infidèles, l'illustre écrivain humaniste renvoie ses concitoyens à l'Affaire qui a tellement remué la conscience civique et fortement secoué les fondements moraux de la société française à la fin du XIX ${ }^{\mathrm{e}}$ siècle. De même qu'à cette occasion Émile Zola lançait son célèbre accusatoire contre l'injustice faite au 
capitaine Dreyfus, victime d'un antisémitisme virulent, de même ses contemporains devaient se dresser contre une guerre qui piétinait toute idée de droit et qui prenait les allures d'une croisade antimusulmane.

Relayant les paroles d'Anatole France une Parisienne, qui s'exprime dans les pages du Gil Blas, vante l'intervention du baron Constant d'Estournelles ${ }^{44}$ en faveur de la paix et fustige la démarche belliqueuse des alliés :

"[...] hélas! vous avez formé de si beaux rêves! Désormais les nations ne devaient plus avoir recours à la force pour discuter leurs droits. Un tribunal devait examiner les faits et rendre un arrêt. Mais les Bulgares, les Serbes, les Grecs refusent de soumettre les questions litigieuses à des experts [...] pour faire une juste opposition à l'ardeur belliqueuse des amis de la guerre, les apôtres de la paix doivent agir. [...] $»^{45}$

Cette imploration est exaucée par les journaux socialistes, ou proches de ceux-ci, qui prennent nettement position contre toute opération militaire. Ainsi, La Petite République du 2 octobre déclare qu': "il est intolérable que les agitateurs de Macédoine continuent indéfiniment à troubler la quiétude universelle, que les petits souverains de Belgrade, de Sofia et d'Athènes persistent à se faire une popularité aux dépens de la paix du monde. »

Les socialistes français, en harmonie avec leurs camarades des pays balkaniques, se prononcent pour la constitution d'une Fédération balkanique seule capable, selon eux, de garantir les droits de tous les peuples de la région. Jean Jaurès, figure de proue de cette campagne et ardent partisan de la paix, informe les lecteurs de L'Humanité que :

"les socialistes de tous les États balkaniques, ceux de Roumanie, de Serbie, de Bulgarie, de Turquie, de Grèce s'organisent pour affirmer leur commune volonté de paix et de réforme. Oui, ils veulent alléger le fardeau qui pèse sur les opprimés. Mais ce n'est pas par des guerres de rapine ou d'orgueil revêtues d'un faux-semblant de philanthropie. C'est en donnant des garanties à tous ceux qui travaillent, c'est en les libérant du joug d'un féodalisme exploiteur qui partout réduit les paysans à n'être que des corvéables et des serfs. C'est en associant les efforts de toutes les races pour une œuvre commune de liberté et de justice sociale »" .

\section{En faveur de l'Empire ottoman : le principe du maintien du statu quo}

Une deuxième catégorie de journalistes et de journaux regroupe ceux qui soit par sympathie envers l'Empire ottoman, soit par sens de pragmatisme politique, insistent sur la nécessité du maintien du statu quo territorial. Parmi eux, des voix isolées comme celle de Pierre Loti dans Le Matin (13 octobre) ou celle de Claude Farrère dans L'Intransigeant, conjurent l'Europe d'intervenir en faveur des Ottomans. Édouard Herriot, sénateur de Rhône et fervent défenseur de la laïcité, avec le Parti radical, va encore plus loin dans son raisonnement, en affirmant que si la Turquie était vaincue, ce seraient la civilisation et l'idéalisme qui, du même coup, seraient cruellement atteints. En même temps, il plaide en faveur du régime des Jeunes-Turcs et il invite ses concitoyens, afin qu'ils puissent juger à son mérite et à ses difficultés la récente révolution turque, à songer à la Révolution française. La Turquie de 1912 se trouve à peu près dans la même situation que la France de 1792, à la veille de Valmy ${ }^{47}$, quand, attaquée de toutes parts, elle essayait d'établir l'ordre nouveau, déclare-t-il.

D'autres journaux, moins favorables a priori au régime des Jeunes-Turcs, en place à Istanbul en ce début de conflit balkanique, se rallient, eux aussi, au principe du respect des frontières de l'Empire ottoman. Ils sont guidés dans ce choix par leur conception de l'intérêt national. Joseph Odelin, dans Le Cri de Paris du 17 octobre, se fait l'exégète de 
cette position. En y exposant son raisonnement, il essaye de convaincre ses lecteurs que l'influence française en Orient dépend tout entière des missions catholiques. Des rapports séculaires et une longue tradition assurent au protectorat français, en Orient et sous le régime ottoman, une prépondérance. Selon sa syllogistique, cette position ne saurait être maintenue si les Bulgares et les Serbes se partageaient la Turquie, «car, le catholicisme n'a pas de pires ennemis dans cette région que les chrétiens orthodoxes et leurs alliés ", souligne-t-il. Cette assertion, qui est également partagée par Paul Cassagnac, directeur de L'Autorité, journal bonapartiste, est contestée par tous ceux qui aspirent à la victoire des Alliés, la considérant, à l'instar de Jollivet, collaborateur du journal Le Cri de Paris, comme un triomphe de la Croix contre le Croissant.

\section{En faveur des alliés : la proposition de la suppression des possessions européennes de l'Empire ottoman}

Les journalistes de La Croix prennent, eux aussi, fait et cause pour la civilisation chrétienne, contre la communauté musulmane. Si leur foi leur dicte les alliances à défendre, leur verdict obéit également à des critères d'intérêt national. Ainsi, dans son article du 26 octobre, le responsable de la politique étrangère s'exclame-t-il :

"[...] regardez les nations en présence. D’un côté la Turquie [...], équipée à l'allemande, instruite par des officiers allemands, encombrée de matériel allemand, la Turquie morceau détaché de la Triplice. De l'autre, la Grèce [...] équipée à la française, instruite par des officiers français; la Serbie dont les munitions françaises font merveille; la Bulgarie créée et mise au monde par la Russie, alliée et amie, la Bulgarie dont les canons français tonnent autour d'Andrinople. Kiamil pacha s'est trompé: ce n'est pas une guerre austro-russe qui se déroule, c'est presque une guerre franco-allemande, Schneider contre Krupp et dont l'enjeu est la prédominance germanique en Orient. $»^{48}$

47 Les journalistes qui souhaitent la défaite ottomane le font à la fois au nom de considérations religieuses et au nom d'appréciations politiques. Les premières renvoient au rejet de l'islam et les secondes à la suprématie de la France en Orient. D'une part, ils pensent qu'une victoire des royaumes chrétiens en Macédoine entraverait les prétentions panislamiques ${ }^{49}$ du régime des Jeunes-Turcs et, d'autre part, qu'un triomphe des armées des alliés, équipées par l'industrie française, sonnerait le glas de l'influence allemande, incapable d'assurer le succès de ses protégés et clients dans cette contrée du continent. Dans ce cas, il s'agirait également d'une revanche, ne fût-ce qu'indirecte, censée effacer, aux yeux de ces journalistes nationalistes, la défaite de 1870 et déblayer le terrain pour la revanche réelle, lors d'une confrontation militaire entre les deux pays, considérée comme imminente.

\section{Conclusion}

En guise de conclusion, on pourrait dire que la représentation spatiale de la Macédoine et la perception de la complexité de la question macédonienne ne diffèrent pas largement d'un journal à l'autre. En revanche, leurs approches et lectures du conflit armé imminent, cristallisent les crispations idéologiques et les divergences politiques qui traversent et travaillent la société française, en ce début du $\mathrm{XX}^{\mathrm{e}}$ siècle. Des nationalistes-militaristes s'opposent à des pacifistes, des russophiles rencontrent des germanophobes, et des anticléricaux se dressent contre les adversaires de la République laïque. On voit s'opposer des journaux et des journalistes proches du Parti 
radical (Le Radical), des socialistes (L'Humanité, La Petite République) ou des libres penseurs, à des journaux et à des journalistes issus des mouvances et des tendances des droites, républicaine (Le Figaro, Le Journal, Le Petit Journal), nationaliste (L'Éclair, Le Cri de Paris) ou catholique (La Croix). Si les uns se caractérisent par leurs discours en faveur d'une résolution pacifique de la question macédonienne, les autres sont, d'une part, animés par des sentiments antiturcs, antimusulmans et antiallemands et, d'autre part par des raisonnements de méfiance à l'égard des Églises orthodoxes des royaumes balkaniques et leurs possibles réactions préjudiciables à la continuité des missions catholiques françaises en Macédoine (Le Cri de Paris, L'Autorité). Parfois, plusieurs voix s'expriment au sein d'un même journal, comme c'est le cas du Cri de Paris et surtout de grands quotidiens comme Le Journal ou Le Matin. Néanmoins, l'unanimité de la presse française sera de nouveau acquise et manifestée quand les journalistes, suite aux premières réussites militaires des alliés, s'interrogeront sur le devenir de la Macédoine, une fois la guerre achevée. Jean Jaurès résume, de la manière la plus éloquente, le point de vue le plus communément partagé par ses collègues, quand il se demande si ces opérations n'aboutiront pas à la conquête et au partage de la Macédoine entre les vainqueurs : «Ô peuples et rois de Monténégro, de Bulgarie, de Serbie, de Grèce [...] qui de vous sera l'élu de Dieu? Mais vous entendrez-vous seulement pour régler le sort de la Macédoine [...] ? Sur la bête déchirée la meute se déchirera. $\|^{50}$ annonce-t-il, de manière prophétique, teintée d'ironie. Quelques mois plus tard, le traité de Bucarest met fin aux hostilités de la Deuxième Guerre balkanique, déclenchée à cause des revendications divergentes et des intérêts conflictuels opposant les alliés d'hier et ayant pour centre le partage des provinces ottomanes de la Macédoine.

\section{NOTES}

1. Pour une présentation générale des enjeux de la question d'Orient, voir Matthew Anderson, The Eastern question: a study in international relations, 1774-1923, London, Macmillan, 1982, Alexander Lyon Macfie, The Eastern question, 1774-1923, London, New York, Longman, 1996 et Stéphane Yerasimos, Questions d'Orient : frontières et minorités des Balkans au Caucase, Paris, Éd. La Découverte, 1993. La plupart des études publiées jusqu'à nos jours s'intéressent surtout aux aspects diplomatiques, militaires et socio-économiques de la question d'Orient et pas tellement à sa perception par les contemporains.

2. Pour une définition des objectifs et de la méthodologie de l'histoire socioculturelle, voir JeanPierre Rioux, Jean-François Sirinelli (dir.), Pour une histoire culturelle, Paris, Éd. du Seuil, 1997 et Pascal Ory, L'histoire culturelle, Paris, PUF, 2004.

3. Pour une périodisation analytique des événements des Guerres balkaniques, voir les tableaux chronologiques dans Egidio Ivetic, Le guerre balcaniche, Bologna, Il Mulino, 2006.

4. En ce qui concerne la déclinaison des sensibilités politiques et la catégorisation des affinités politiques des journaux français de l'époque, voir la typologie établie par Christophe Charle dans, Le siècle de la presse, 1830-1939, Paris, Éditions du Seuil, 2004 (notamment le chapitre intitulé «Journaux et journalistes dans les années 1880-1900). 
5. Pour une présentation des différentes tendances de la droite en France, voir René Rémond, Les droites en France, Paris, Aubier, 1990 (1982).

6. Jean-Jacques Brousson, « Courrier de Paris », Gil Blas, 17 octobre 1912.

7. Sur les méthodes de l'enquête sociologique inaugurées et pratiquées par les disciples de l'école leplaysienne, voir Bernard Kalaora, Les inventeurs oubliés : Le Play et ses continuateurs aux origines des sciences sociales, Paris, Champ Vallon, 1989.

8. Azambuja Gabriel, Le conflit des races en Macédoine, d'après une observation monographique, Paris, 1904, p. 3.

9. «La guerre d'Orient », Le Temps, 16 octobre 1912.

10. Léo Clarétie, « Choses de Bulgarie », Le Petit Parisien, 12 octobre 1912.

11. Sur les références médiévales des revendications irrédentistes des pays balkaniques, à la fin du XIX ${ }^{\mathrm{e}}$ siècle et au début du XX $\mathrm{X}^{\mathrm{e}}$ siècle, voir Alexandru Madgearu, The wars of the Balkan Peninsula, their medieval origins, Lanham, Scarecrow Press, 2008.

12. Le traité de San Stefano mettait fin à la guerre russo-turque de 1877 et attribuait à la Bulgarie la majeure partie de la région géographique de la Macédoine.

13. Nacertanje : projet, dessein en serbe. Il indique le programme national des Serbes rédigé par Ilija Garašanin en 1844. Les buts de la politique extérieure de la Serbie y sont énoncés et puisent leur inspiration dans le souvenir de l'empire de Dušan au XIV ${ }^{\mathrm{e}}$ siècle.

14. André Morizet, « Pierre Ier de Serbie », L'Humanité, 8 octobre 1912.

15. L'idée de ce projet expansionniste fut diffusée par le Premier ministre grec Jean Kolettis en 1844, en antidote comme le soutient Georges Castellan aux dysfonctionnements multiples de la vie politique interne du royaume grec de cette époque.

16. Le Matin, 26 octobre 1912.

17. Jules Arren, « Le casse-tête macédonien », L'Éclair, 14 octobre 1912.

18. Idem.

19. La Macédoine constituait, à la fin du XIX et au début du XX $X^{\mathrm{e}}$ siècle, une région géographique dans laquelle se confrontaient les intérêts géostratégiques et économiques divergents entre la politique de Drang nach Osten de l'Empire austro-hongrois et de l'Allemagne d'une part, et la volonté de l'Empire tsariste pour un accès à la mer Égée d'autre part.

20. « La Macédoine » par R.T., La Croix, 19 octobre 1912.

21. Le 20 juillet (2 août) 1903, jour de la Saint-Élie (Ilinden), l'Organisation révolutionnaire intérieure macédonienne (ORIM) lance une révolte armée contre l'autorité ottomane dans le vilayet de Monastir (Bitola). Au départ, les insurgés remportent des succès et réussissent à proclamer une République autonome, la République de Kruševo, avant que leur insurrection ne soit brutalement réprimée. L'ampleur des représailles est pour l'empereur François-Joseph et le tsar Nicolas II une occasion favorable pour intervenir dans l'organisation politique de la Macédoine. Après leur rencontre au château de Mürzsteg, ils imposent au sultan un programme de réformes, selon lequel l'inspecteur général ottoman serait assisté en Macédoine de deux " agents civils ", l'un autrichien et l'autre russe. De plus, la région de la Macédoine serait divisée en secteurs de police, dont le contrôle était confié à chacune des cinq grandes puissances de l'époque : l'Angleterre, la France, l'Italie, l'Autriche et la Russie. Cette tutelle administrative resta en vigueur jusqu'à l'avènement au pouvoir des Jeunes-Turcs en 1908. Pour une étude analytique des activités de l'ORIM, voir Dunkan M. Perry, The politics of terror: the Macedonian liberation movements, 1893-1903, Durham, Duke University Press, 1988.

22. Le Figaro, $1^{\mathrm{er}}$ octobre 1912.

23. Jean Jaurès, « La crise balkanique, pas d'illusion », L'Humanité, 7 octobre 1912.

24. Pour le rôle primordial joué par la Fédération socialiste ouvrière des Balkans dans la coordination des initiatives antimilitaristes dans les Balkans pendant la période des Guerres balkaniques, voir Paul Dumont, «La Fédération socialiste ouvrière de Salonique à l'époque des guerres balkaniques ", East European Quaterly, 1980, vol. XIV, nº 4, pp. 383-410. 
25. Marcel Cachin, « Lourd matérialisme », L'Humanité, 15 octobre 1912.

26. « La mobilisation balkanique », Le Journal des Débats, 2 octobre 1912.

27. «La Guerre d'Orient par Tout-Paris, Bloc-notes parisien », Le Gaulois, 13 octobre 1912.

28. « La Turquie réduite aux abois », par R.T, La Croix, 3 octobre 1912.

29. Cette approche pourrait nous faire penser à la division de notre planète en zones ou aires «civilisationnelles» potentiellement conflictuelles décrites par Samuel Huntington dans son ouvrage Le choc des civilisations (traduction française) Paris, O. Jacob, 1997.

30. Louis Dubreuilh, « Ni croix ni croissant », L'Humanité, 8 octobre 1912.

31. Pour une synthèse des débats autour de la notion de civilisation, ainsi que pour une présentation de la place occupée par le discours racial dans des disciplines telles que la sociologie ou l'ethnologie, en France, au tournant du XX $\mathrm{X}^{\mathrm{e}}$ siècle, voir Laurent Mucchielli, La découverte du social, naissance de la sociologie en France, Paris, La Découverte, 1998.

32. André Chéradame, "La Guerre des Balkans et les intérêts de la France ", Le Petit Journal, 17 octobre 1912.

33. « Pourquoi la guerre ?», Le Journal, 19 octobre 1912.

34. Louis Dubreuilh, « Ni croix ni croissant ", L'Humanité, 8 octobre 1912.

35. « Pour Dieu Pour la France », par R.T., La Croix, 26 octobre 1912.

36. «Ce que disent les journaux », La Croix, 26 octobre 1912.

37. Voir Claude Bellanger (dir.) Histoire générale de la presse française, tome III, entre 1871 et 1940, Paris, PUF, 1972.

38. Sur les pratiques de financement de la presse française au début du $\mathrm{XX}^{\mathrm{e}}$ siècle, voir Félicien Challaye, «Politique internationale et journalisme d'affaires » dans Revue du mois, 10 juin 1911.

39. Le sujet des différentes étapes et expressions du nationalisme français pendant la période 1870-1914 a été amplement traité dans la bibliographie francophone et anglo-saxonne. Parmi les nombreuses références bibliographiques, on pourrait consulter avec intérêt, celles de Jean-Jacques Becker, Stéphane Audoin-Rouzeau, La France, la nation, la guerre, 1850-1920, Paris, Sedes, 1995, Eugen Weber, The nationalist revival in France, 1905-1914, Berkeley, University of California Press, 1959 et Zeev Sternhell, Maurice Barrès et le nationalisme français, Bruxelles, Éd. Complexe, 1985 (1972).

40. Sur la polémique suscitée par l'élaboration de la loi des trois ans et la place que celle-ci a occupée dans les débats publics en France, voir Miller Paul, From Revolutionaries to Citizens: antimilitarism in France 1870-1914, Durham, London, Duke University Press, 2002 et Krumreich Gerd, Armaments and Politics in France on the eve of the First World War. The introduction of the threeyear conscription, 1913-1914, Dover, Berg Publishers, 1984.

41. Voir Jacqueline Lalouette, La République anticléricale, XIX-XX ${ }^{e}$ siècle, Paris, Éd. du Seuil, 2002.

42. Jean Jaurès, « Réacteurs dupes » L'Humanité, 3 novembre 1912.

43. Gil Blas, 5 octobre 1912.

44. Fervent opposant à la politique coloniale et à l'augmentation des dépenses militaires, il était également favorable à un rapprochement franco-allemand. Ses efforts pour la promotion de l'idée d'un arbitrage pacifique entre les nations lui ont valu le prix Nobel en 1909. Pour plus d'informations concernant sa vie et son œuvre, voir Laurent Barcelo, Paul d'Estournelles de Constant : l'expression d'une idée européenne, Paris, L'Harmattan, 1995.

45. « Billet d'une Parisienne à M. d'Estournelles de Constant », Gil Blas, 11 octobre 1912.

46. Jean Jaurès, « Pour la paix » L'Humanité, 8 octobre 1912.

47. Édouard Herriot, « France et Turquie », Le Journal, 20 octobre 1912.

48. « Pour Dieu, Pour la France », par R.T., La Croix, 26 octobre 1912.

49. Ces prétentions étaient conçues comme des véritables menaces de pays qui comme la France, l'Angleterre ou la Russie, contrôlaient des populations ou des régions avec d'importantes communautés musulmanes.

50. L'Humanité, 2 octobre 1912. 


\section{RÉSUMÉS}

Comment la presse française, en trois familles idéologiques distinctes, présente la question macédonienne en 1912.

L'objectif de cette communication est d'étudier comment la presse française aborda la question macédonienne au commencement de la première guerre balkanique. Une première catégorie de journaux (socialistes, syndicalistes, anarchistes, anticléricaux) se prononce pour la paix et soutient soit le maintien du statu quo territorial, soit la création d'une fédération balkanique, en dénonçant en même temps le caractère expansionniste des projets des alliés balkaniques. Une deuxième catégorie (nationalistes de droite, antirépublicains) adopte la rhétorique officielle des royaumes balkaniques qui justifiaient leur mobilisation au nom de la défense des droits de leurs coreligionnaires dans l'Empire ottoman et notamment en Macédoine. La plupart de ces plumes ont trempé d'une part dans la haine du Turc et du musulman et sont inspirées d'autre part, par leur volonté revancharde contre l'Allemagne qui aurait équipé l'armée ottomane alors que l'industrie française aurait doté les alliés balkaniques. Enfin, une dernière catégorie (catholiques, bonapartistes) considère que le bouleversement du statu quo territorial au profit des royaumes orthodoxes serait préjudiciable pour les intérêts économiques et culturels français.

French newspapers comment the Macedonian question at the dawn of the Balkan Wars.

The aim of this paper is to study how the French newspapers dealt with the Macedonian question at the beginning of the Balkan Wars. A first group (socialists, trade-unionists, anarchists, anticlerical), backs up a peaceful settlement of the dispute and is in favor of the maintenance of the status quo or the creation of a Balkan federation. It is also denouncing the expansionist character of allies' projects. A second group (right nationalists, antirepublicans) adopts the official rhetoric of the Balkan kingdoms, justifying their mobilization in the name of the defense of Christian communities in the Ottoman Empire, notably in the region of Macedonia. The majority of these articles are instilled with the hate of the Turk and the Muslim, and they are inspired by their authors' will to take revenge against Germany, whose military industry had equipped the ottoman army whereas the allies' war machines had been fabricated in France.

Finally, a last category (catholics, bonapartists) considers that French economical and cultural interests would be jeopardized in the case of a victory of the Orthodox Balkan kingdoms

\section{INDEX}

Thèmes : Histoire, Sciences politiques

Index chronologique : guerres balkaniques (1912-1913)

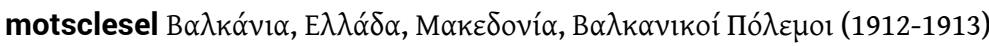

motsclestr Balkanlar, Yunanistan, Makedonya, Balkan Savaşları (1912-1913)

Mots-clés : fédération balkanique, ORIM/VRMO, presse française, San Stefano, Touranien

motsclesmk БАЛКАНОТ, ГРЦИЈА, МАКЕДОНИЈА

Index géographique : Balkans, Grèce, Macédoine

Keywords : French press, Balkan federation, Politics, Balkans, Greece, Macedonia 
AUTEUR

NICOLAS PITSOS

Enseignant à l'ICES, Doctorant CREE-CEB EA 4513 